\title{
BMJ Open Assessment of potential predictors of calretinin and mesothelin to improve the diagnostic performance to detect malignant mesothelioma: results from a population-based cohort study
}

\author{
Swaantje Casjens, ${ }^{1}$ Daniel G Weber, ${ }^{1}$ Georg Johnen, ${ }^{1}$ Irina Raiko, ${ }^{1}$ Dirk Taeger, ${ }^{1}$ \\ Carmen Meinig, ${ }^{1}$ Susanne Moebus, ${ }^{2}$ Karl-Heinz Jöckel, ${ }^{2}$ Thomas Brüning, ${ }^{1}$ \\ Beate Pesch ${ }^{1}$
}

To cite: Casjens S, Weber DG, Johnen G, et al. Assessment of potential predictors of calretinin and mesothelin to improve the diagnostic performance to detect malignant mesothelioma: results from a populationbased cohort study. BMJ Open 2017;7:e017104. doi:10.1136/ bmjopen-2017-017104

- Prepublication history for this paper is available online. To view these files, please visit the journal online (http://dx.doi. org/10.1136/bmjopen-2017017104)

SC, DGW, TB and BP contributed equally.

Received 31 March 2017 Revised 4 August 2017 Accepted 9 August 2017

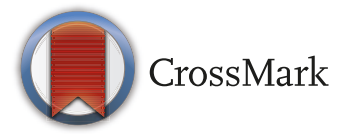

${ }^{1}$ Institute for Prevention and Occupational Medicine of the German Social Accident Insurance, Ruhr-Universität Bochum (IPA), Bochum,

Germany

${ }^{2}$ Institute of Medical Informatics, Biometry and Epidemiology, University Duisburg-Essen, Essen, Germany

Correspondence to Dr Swaantje Casjens; casjens@ipa-dguv.de

\section{ABSTRACT}

Objectives Mesothelin and calretinin are blood-based markers for malignant mesothelioma. The objective of this study was to analyse the markers in plasma samples from cancer-free men and to identify factors influencing their concentrations to minimise false-positive test results when using these markers for the early detection of malignant mesothelioma.

Setting The present analyses used data and archived blood samples of the population-based Heinz Nixdorf Recall Study among elderly people collected from 2011 to 2014.

Participants A total of 569 men (median age 70 years) without a malignant disease at the time of blood sampling were selected for these analyses.

Primary and secondary outcome Mesothelin and calretinin concentration in plasma samples.

Results We observed 24 mesothelin concentrations $\geq 1.5 \mathrm{nM}$ (specificity $95.8 \%, 95 \% \mathrm{Cl} 93.8 \%$ to $97.2 \%$ ) and 34 calretinin concentrations $\geq 1.0 \mathrm{ng} / \mathrm{mL}$ (specificity $94.0 \%$, $95 \% \mathrm{Cl} 91.7 \%$ to $95.7 \%$ ). Only five men had both markers above these cut-offs. Renal dysfunction and hypertension were major predictors of elevated mesothelin in addition to age. Regarding calretinin, the effect of renal dysfunction was slightly weaker and hypertension was not associated with increased concentrations. However, a diagnosis of cancer after blood collection and bronchial asthma were associated with positive calretinin results.

Conclusions The combined determination of mesothelin and calretinin results in only few false-positive marker tests. Both markers are mainly influenced by renal dysfunction. The determination of cystatin $\mathrm{C}$ concentrations may be informative when interpreting the test results.

\section{INTRODUCTION}

Malignant mesothelioma is an aggressive cancer of the serous membranes with an increasing incidence worldwide. ${ }^{1}$ Mesothelioma shows a latency period up to 40 years and median survival after diagnosis is between

\section{Strengths and limitations of this study}

- This is the first study that extensively examines potential predictors associated with positive test results of blood-based markers for mesothelioma in plasma samples of cancer-free participants in a large population-based cohort study.

- Strong determinants of elevated marker concentrations were identified in order to improve the specificity of calretinin and mesothelin for the early detection of malignant mesothelioma.

- Due to very high overall specificities of mesothelin and calretinin of $95 \%$, the statistical analyses on predictors of false-positive tests were based on small numbers.

- The study population was enrolled from the general population and not a cohort of asbestos workers with a higher prevalence of asbestosis.

- The study focused on men only because the incidence of mesothelioma is significantly higher in men than in women. Hence, possible gender differences could not be investigated.

9 and 13 months, depending on treatment, ${ }^{2}$ because symptoms commonly occur only at late stages of this cancer. Thus, the diagnosis of mesothelioma at early stages might be a promising opportunity to improve therapy.

Up to date, the most prominent bloodbased marker for mesothelioma is mesothelin. As recently shown in a meta-analysis, mesothelin as individual marker is characterised by a specificity of $89 \%(95 \%$ CI $86 \%$ to $91 \%$ ) and a sensitivity of $58 \%$ (95\% CI $54 \%$ to $62 \%$ ) for the discrimination of patients with mesothelioma from asbestos-exposed subjects. ${ }^{3}$ One of the best immunohistochemical markers for mesothelioma is calretinin. ${ }^{4}$ Furthermore, it was shown that calretinin concentrations were elevated in the blood 
of patients with diagnosed mesothelioma compared with control groups. ${ }^{5}$ Calretinin as individual blood-based marker reached $71 \%$ sensitivity at a fixed specificity of 95\% for the discrimination of patients with mesothelioma from asbestos-exposed subjects. ${ }^{6}$

However, in screening with tumour markers a very high specificity is desirable to avoid false-positive tests in cancerfree subjects. ${ }^{7}$ Thus, there is an urgent need to identify parameters influencing the marker concentrations in subjects without the malignant disease. For mesothelin it is well known that renal failure could lead to increased marker concentrations, ${ }^{8-10}$ but less is known about the effect on calretinin. To improve the use of markers as screening tools for the early detection of cancer, strong determinants of false-positive tests in a cancer-free population have to be considered. ${ }^{10}$

The aim of this study was to analyse mesothelin and calretinin in plasma samples from a large group of cancerfree men with a comprehensive set of auxiliary data in order to identify predictors of positive tests to improve the specificity of the markers for the early detection of malignant mesothelioma.

\section{METHODS}

\section{Ethics statement}

The study was approved by the ethical commission of the Medical Faculty of the University Duisburg-Essen (approval number 11-4678). All participants gave their written informed consent.

\section{Study population}

The study population consisted of 569 men from the second follow-up survey (2011-2014) of the Heinz Nixdorf Recall Study (HNRS) within the framework of AeKo ('Arbeitsmedizinische Forschung in epidemiologischen Kohortenstudien'-Occupational medical research in epidemiological cohort studies). ${ }^{11}$ HNRS is a prospective population-based cohort study in the Ruhr area, an urban conglomeration of industrial cities in Germany. Its rationale, design and conduct have been previously described. ${ }^{12}$ For this analysis, subjects did not suffer from a malignant disease at the time of blood collection.

\section{Determination of mesothelin and calretinin}

For measurement of mesothelin and calretinin peripheral blood was collected from each participant in $9.0 \mathrm{~mL}$ S-Monovette EDTA gel tubes (Sarstedt, Nümbrecht, Germany) and centrifuged at $2000 \mathrm{x}$ g for $10 \mathrm{~min}$. Plasma was separated from the cellular fraction and frozen immediately.

Plasma mesothelin $(\mathrm{ng} / \mathrm{mL})$ was measured using the Human Mesothelin Quantikine ELISA kit (R\&D Systems, Wiesbaden-Nordenstadt, Germany) according to the manufacturers' instructions. Results were converted to nanomolar using the equation $\mathrm{nM}=\mathrm{xng} / \mathrm{mL} / 32.175$. Marker results were marked as positive if mesothelin concentrations were $\geq 1.5 \mathrm{nM}$ (cut-off). The intra-assay coefficient of variation (CV) was $3.6 \%$ and the inter-assay CV was $5.7 \%$.

Plasma calretinin $(\mathrm{ng} / \mathrm{mL})$ was measured as described previously. ${ }^{5}$ Marker results were marked as positive if calretinin concentrations were $\geq 1.0 \mathrm{ng} / \mathrm{mL}$ (cut-off). For the calretinin assay the intra-assay $\mathrm{CV}$ was $6.3 \%$ and the inter-assay CV was $6.4 \%$.

A high stability of mesothelin and calretinin in plasma and serum was revealed regarding frozen storage as well as repeated freeze/thaw cycles. ${ }^{58} 813$

\section{Potential predictors of positive marker tests}

We used information on potential predictors of positive tests from the questionnaire (age, smoking status, hypertension, chronic diseases such as asthma and diabetes mellitus, and medication). We further included follow-up

\begin{tabular}{|c|c|c|c|c|}
\hline Blood parameter & Standard value & $\mathbf{n}$ & Median & IQR \\
\hline Brain natriuretic peptide $(\mathrm{pg} / \mathrm{mL})$ & $<200$ & 569 & 26.7 & $13.8-54.9$ \\
\hline C-reactive protein (mg/dL) & $0-0.3$ & $564^{*}$ & 0.13 & $0.07-0.29$ \\
\hline Creatinine (mg/dL) & $0.9-1.3$ & 569 & 1.16 & $1.06-1.27$ \\
\hline \multirow[t]{3}{*}{ Cystatin C (mg/L) } & 19-65 years: $0.53-0.95$ & $568^{*}$ & 0.86 & $0.75-0.99$ \\
\hline & 66-74 years: $0.6-1.3$ & & & \\
\hline & $\geq 75$ years: $0.7-1.47$ & & & \\
\hline Fibrinogen (mg/dL) & $210-400$ & $567^{*}$ & 328 & $281-380$ \\
\hline Hemoglobin A1c (\%) & $4-6$ & $567^{*}$ & 5.9 & $5.6-6.2$ \\
\hline \multirow[t]{3}{*}{ Haemoglobin (g/dL) } & 16-70years: $13.7-17.2$ & 569 & 14.9 & $14.1-15.5$ \\
\hline & $71-75$ years: $12.1-17.6$ & & & \\
\hline & $76-81$ years: $11.8-17.5$ & & & \\
\hline Lactate dehydrogenase (U/L) & $100-247$ & $568^{*}$ & 177 & $160-196$ \\
\hline Red blood cells $\left(\times 10^{12} \mathrm{~L}\right)$ & $4.5-5.6$ & 569 & 4.8 & $4.55-5.05$ \\
\hline
\end{tabular}

${ }^{\star}$ Blood parameters could not be determined for all 569 subjects. 
Table 2 Characteristics of 569 men of the Heinz Nixdorf Recall Study

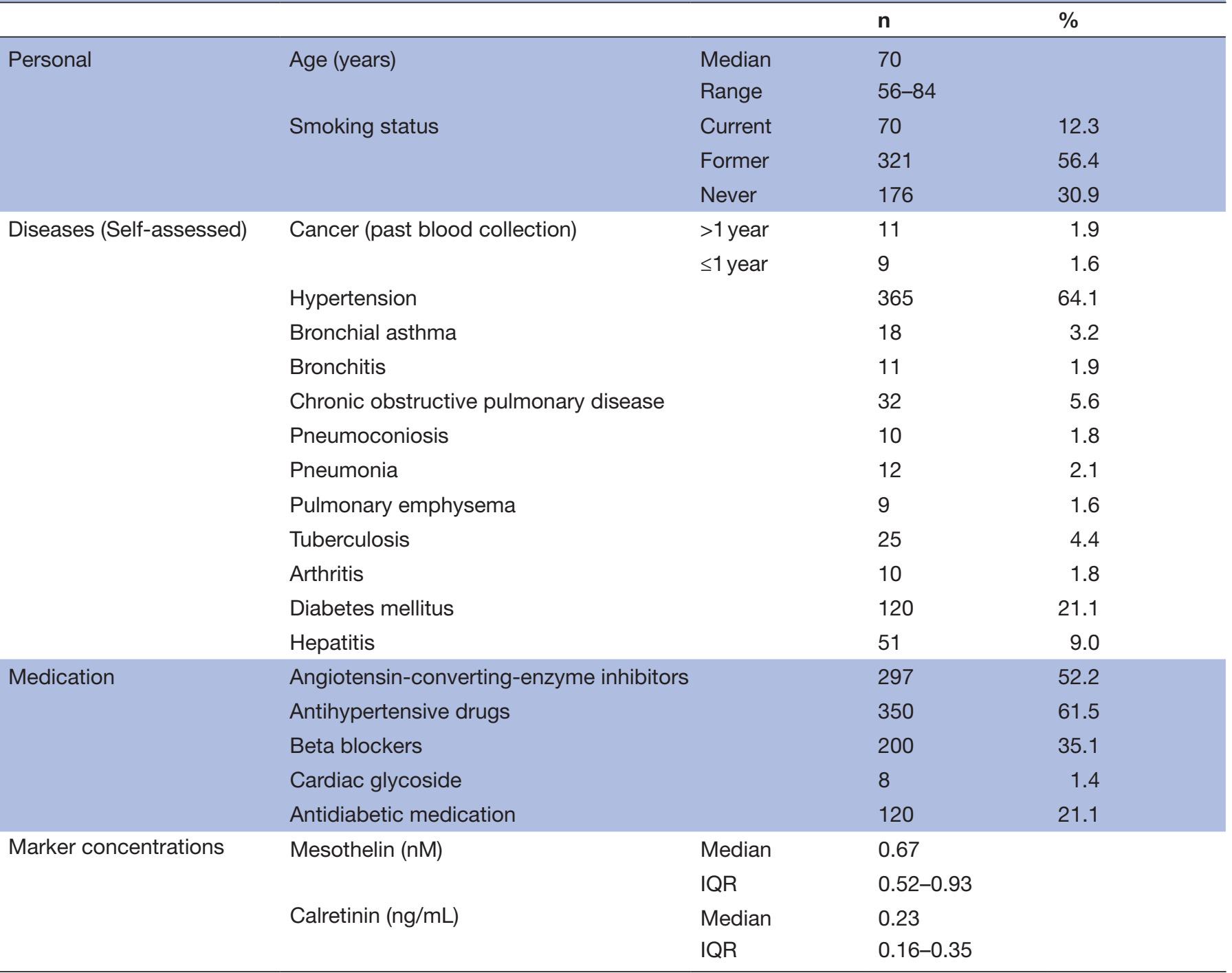

information on the development of cancer and data on miscellaneous blood parameters at time of blood sampling. The distribution of blood parameters in the study population and standard values are given in table 1 . All blood analyses were performed in the central laboratory of the University Hospital Essen with standard methods as formerly described. ${ }^{14}$

\section{Statistical analysis}

All calculations were done using SAS V.9.4 (SAS Institute). Median and inter-quartile range (IQR) were used to describe the distribution of continuous variables. Spearman's correlation coefficients $\left(r_{s}\right)$ and $p$ values were used to describe rank correlations between variables.

Prevalence ORs (PORs) with 95\% CIs were estimated to assess the risk of marker concentrations being above the cut-off. Potential predictors were age, smoking status (never, former current), 11 different diseases (yes, no), development of cancer after blood sampling, intake of five different medications within the last 7 days before examination (yes, no) and 36 laboratory parameters (within, below or above standard values). First, we estimated PORs with univariate logistic regression models to identify potential influencing variables. Based on knowledge from literature and preliminary work, variables were assorted to corresponding disease groups, that is, malignant (diagnosis after blood collection), renal and heart diseases. Subsequently, for each marker separately, multiple regression models with one parameter from each disease group based on the univariate models were developed. As representative of the disease group the parameter with the strongest effect was selected.

\section{RESULTS}

\section{Study population}

Table 2 depicts the characteristics and marker results of 569 men from HNRS. The median age of the study population was 70 years (range: 56-84years). A large fraction of the participants were former smokers (56.4\%), 176 
participants $(30.9 \%)$ were non-smokers and 70 participants $(12.3 \%)$ were current smokers.

After blood collection cancer was diagnosed in 20 participants, where 9 cases received a diagnosis within 12 months. The most frequent disease was hypertension with 365 cases $(64.1 \%)$, followed by diabetes mellitus with 120 cases $(21.1 \%)$. Hence, antihypertensive drugs were commonly used $(\mathrm{n}=350,61.5 \%)$, and there was a very high correlation between hypertension and the intake of heart medications. Nearly every participant with hypertension took antihypertensive drugs (95.9\%), and only every other took beta blockers $(54.2 \%)$.

\section{Blood parameters}

The median concentrations of all blood parameters were within the standard values (table 1 ). Median mesothelin concentration was $0.67 \mathrm{nM}$ (IQR $0.52-0.93 \mathrm{nM}$ ) and median calretinin concentration $0.23 \mathrm{ng} / \mathrm{mL}$ (IQR $0.16-0.35 \mathrm{ng} / \mathrm{mL}$ ) (table 2). Positive mesothelin results $(\geq 1.5 \mathrm{nM})$ were observed for 24 subjects $(4.2 \%)$ and positive calretinin results $(\geq 1.0 \mathrm{ng} / \mathrm{mL})$ for 34 subjects $(6.0 \%)$. Hence, the specificities for mesothelin and calretinin were $95.8 \%$ (95\% CI $93.8 \%$ to $97.2 \%$ ) and $94.0 \%$ (95\% CI $91.7 \%$ to $95.7 \%$ ) for the whole study population, respectively. Five men had elevated concentrations of both markers, resulting in a specificity of $99.1 \%$ (95\% CI $97.9 \%$ to $99.7 \%$ ).

The Spearman correlations between mesothelin, calretinin, age and blood parameters are presented in (table $3)$. Calretinin and mesothelin were positively correlated $\left(r_{\mathrm{s}}=0.18, \mathrm{p}<0.001\right)$. The strongest correlation with $\mathrm{r}_{\mathrm{s}}=0.55$ $(\mathrm{p}<0.001)$ was observed between cystatin $\mathrm{C}$ and creatinine, both being markers of renal function. The concentrations of both markers increased by age, but age was also positively correlated with most of the blood parameters.

\section{Potential predictors of positive test results for mesothelin and calretinin}

Table 4 depicts the results of the univariate logistic regression models for predictors of mesothelin and calretinin concentrations above the cut-off. Cystatin C, creatinine, brain natriuretic peptide (BNP) and haemoglobin are associated with renal dysfunction. Cystatin $\mathrm{C}$ showed a POR of 20.8 (95\% CI 8.48 to 50.8) for mesothelin and 3.48 (95\% CI 1.34 to 9.02) for calretinin. Creatinine, BNP, haemoglobin, haematocrit, and the amount of red blood cells showed an impact only on mesothelin. Furthermore, hypertension and the intake of heart medication had an impact on increased POR only for mesothelin (13.7 (95\% CI 1.83 to 101.9 ) and 15.3 (95\% CI 2.06 to 114.4 ), respectively). The intake of beta blockers was associated with both markers.

Age showed a significantly increased POR (1.09, 95\% CI 1.02 to 1.17) for mesothelin but not for calretinin. Cancer diagnosed after blood collection showed an POR of 4.33 (95\% CI 1.36 to 13.7) for calretinin. In contrast, mesothelin was not increased in participants with a diagnosis of cancer after blood collection. Furthermore, $\mathrm{C}$ reactive protein and fibrinogen were associated with mesothelin but not calretinin. Bronchial asthma was a predictor of elevated calretinin. Bronchitis and increased hemoglobin A1c (HbA1c), a marker associated with diabetes mellitus, showed an impact on mesothelin. Several other parameters showed an increased POR for mesothelin (eg, alkaline phosphatase) but were based on very small numbers, whereas calretinin was not affected by other parameters (data not shown).

Table 5 shows the results of the multiple logistic regression models in the whole study population. Regarding the risk estimates for a positive mesothelin test, the PORs for cystatin C, hypertension and age were 11.0 (95\% CI 4.02 to 30.0 ), 8.41 (95\% CI 1.07 to 66.0 ) and 1.08 (95\% CI 1.00 to 1.17 ), respectively. Regarding calretinin, cystatin $\mathrm{C}$, cancer diagnosed after blood collection and bronchial asthma showed an impact on the marker concentration with a POR of 4.03 (95\% CI 1.49 to 10.9$), 4.98$ (95\% CI 1.51 to 16.5 ) and 5.19 (95\% CI 1.54 to 17.4 ), respectively. Hypertension did not affect calretinin (POR 1.05, 95\% CI 0.49 to 2.27 ).

\section{DISCUSSION}

Commonly, mesothelioma is diagnosed at late stages of the disease when symptoms like coughing, chest pain and difficulty breathing occur. Principally, tumour markers might facilitate the detection of cancer at early stages when clinical symptoms have not yet occurred. Mesothelin and calretinin are two candidates to serve as reliable blood-based markers for the early detection of mesothelioma. ${ }^{346}$ Generally, the diagnosis of cancer at early stages, when clinical symptoms have not yet occurred, appears to be a promising opportunity to improve therapeutic outcome, ideally resulting in a decreased mortality. The treatment of early stages in combination with the new therapies that are on the horizon could lead to improvements in overall survival of patients with mesothelioma. ${ }^{15-19}$

In screening, false-positive tests should be avoided in cancer-free individuals. ${ }^{720}$ However, knowledge is limited regarding the influence of benign diseases and blood parameters as potential confounders of mesothelin and calretinin, leading to increased marker concentrations in subjects without malignant mesothelioma.

This is the first study that extensively examines potential influencing parameters associated with mesothelin and calretinin concentrations above the cut-off in plasma samples of cancer-free participants in a large population-based cohort study. In this population-based cohort, we observed overall specificities of mesothelin and calretinin of about $95 \%$. Only five men had both markers above the cut-offs, which would indicate a specificity of $99.1 \%$ (95\% CI $97.7 \%$ to $99.7 \%$ ) in the general population. In a recent study, we were able to demonstrate that a combination of mesothelin and calretinin was able to improve the performance of mesothelin alone. ${ }^{6}$ The specificity in the target population of asbestos-exposed 


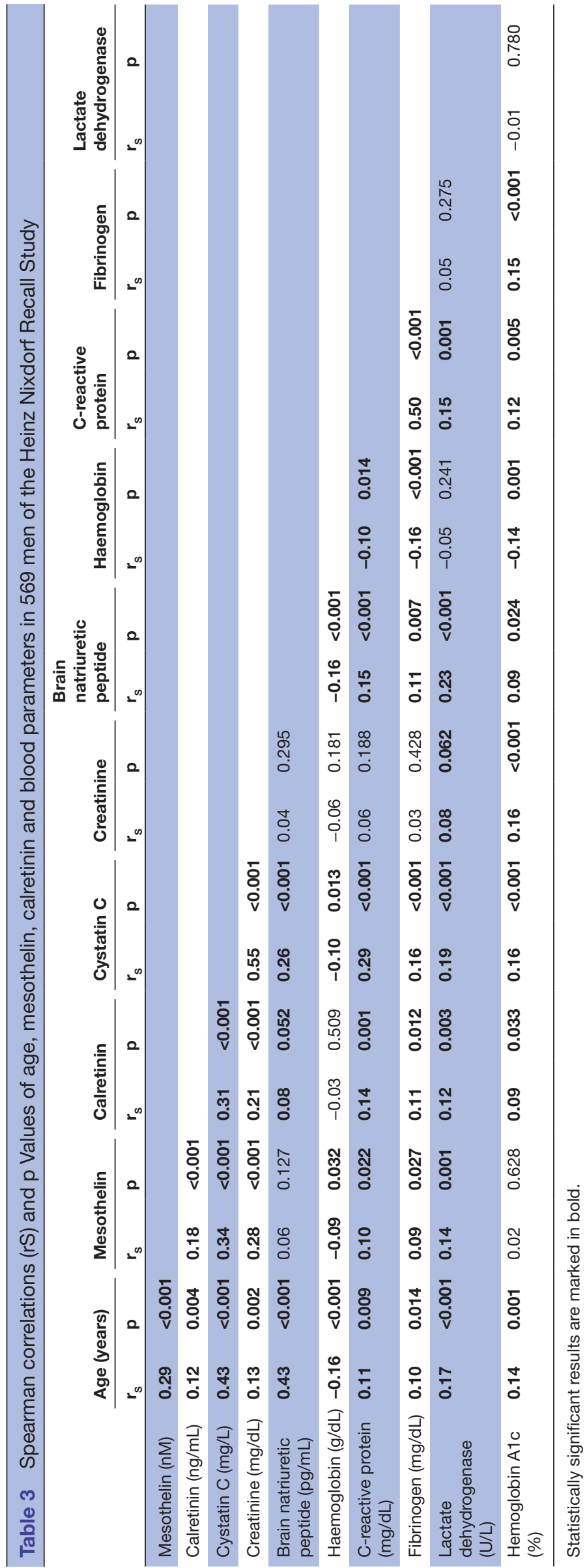

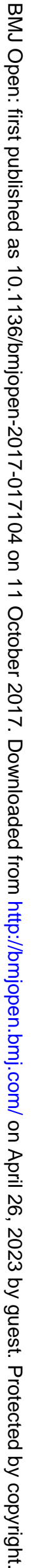




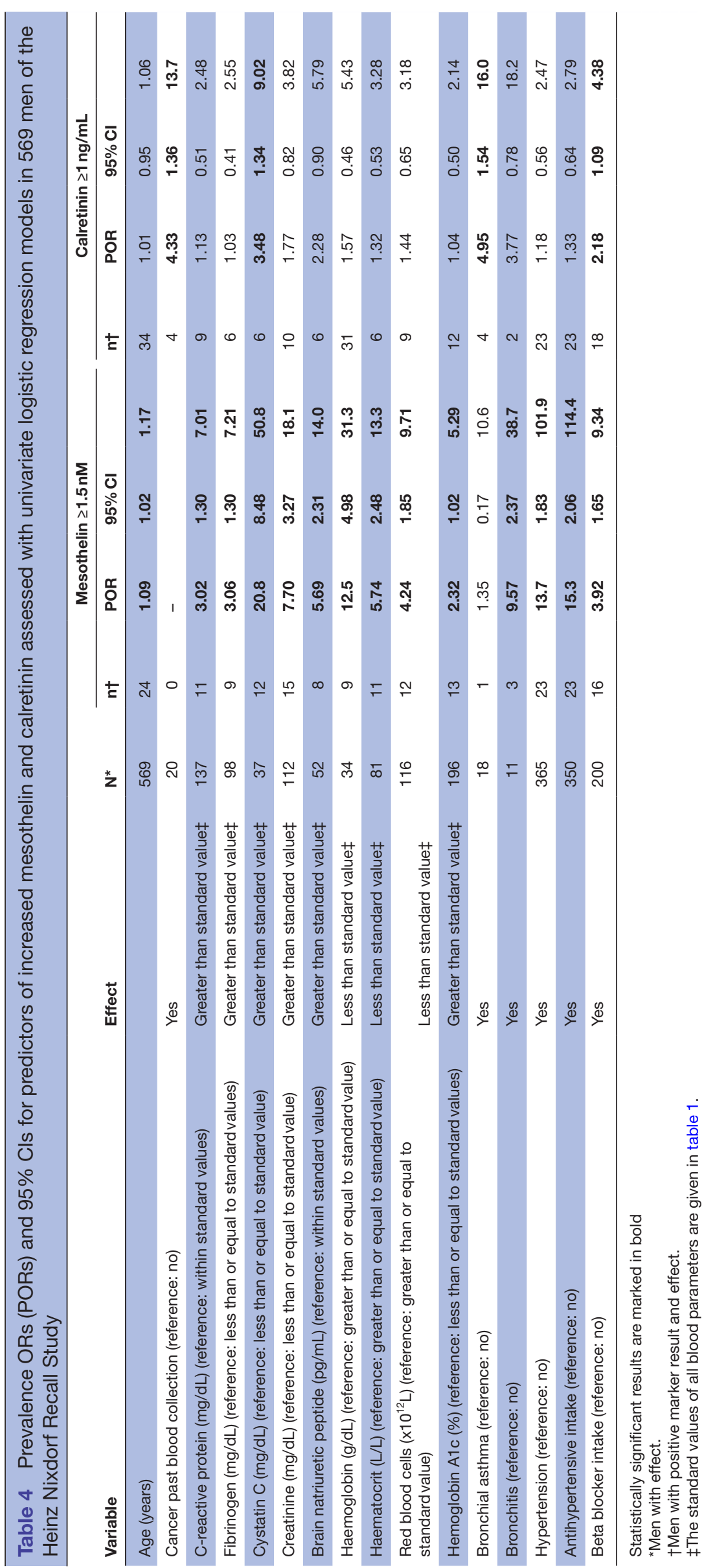




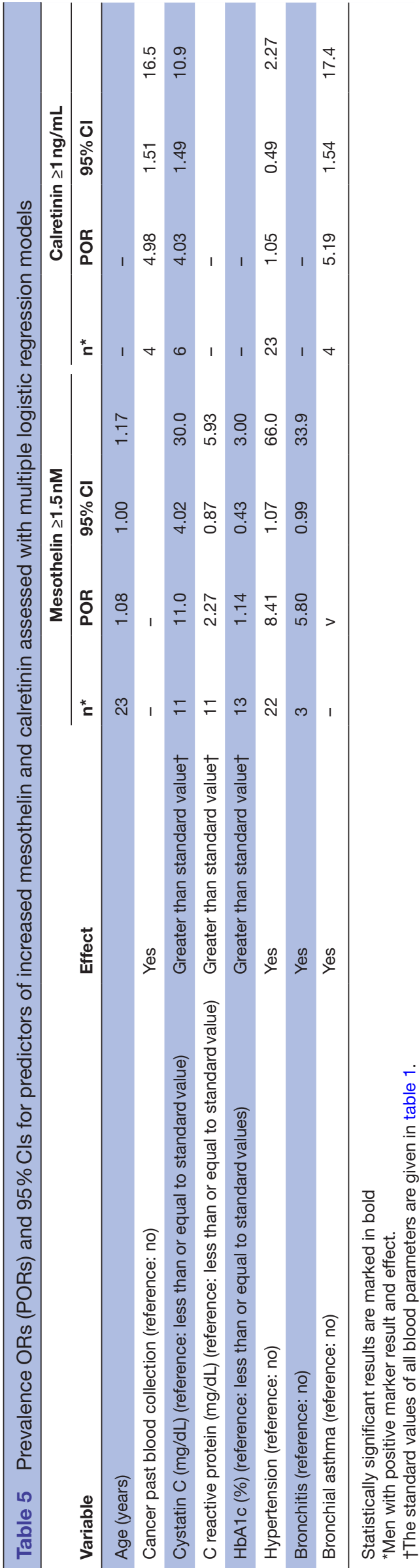

workers, including cases with asbestosis, is likely lower as shown in a meta-analysis. ${ }^{3}$

An advantage of this population-based cohort is the wealth of data acquired, which cannot be assembled in clinical routine. ${ }^{7}$ However, the study focused on men because in general the reported incidences of mesothelioma are significantly higher for men than women. ${ }^{21} 22$ This might be a result of an increased occupational exposure to asbestos among men. ${ }^{23}$ Hence, it was not the aim of this study to determine whether gender differences exist regarding the association between calretinin, mesothelin and the potential confounding factors investigated. Furthermore, despite the large study population the statistical analyses on predictors of false-positive tests were based on small numbers which resulted from the high overall specificities of mesothelin and calretinin of $95 \%$.

In this study, renal dysfunction as a well-known confounder was confirmed for mesothelin. An influence of renal dysfunction was also identified for calretinin although weaker. The glomerular filtration rate was shown to be a confounder of mesothelin in serum ${ }^{9}$ and similar results were observed for the association of creatinine and mesothelin. ${ }^{10}$ Here, we showed that increased cystatin $\mathrm{C}$ values correlated with increased mesothelin and calretinin concentrations in plasma. Notably, the impact of cystatin $\mathrm{C}$ on mesothelin seems to be stronger than on calretinin. For the assessment of renal dysfunction, cystatin $\mathrm{C}$ might be superior to creatinine because it is not influenced by age, gender and muscle mass. ${ }^{24} 25$ For the use of mesothelin and calretinin in diagnostics or early detection, the additional assessment of cystatin $\mathrm{C}$ might be meaningful to improve specificity.

Hypertension is a common cause but also a consequence of kidney failure ${ }^{26}$ and was already mentioned as potential confounder of mesothelin. ${ }^{8}$ In this study, men with hypertension showed an eightfold higher chance of increased mesothelin concentrations in comparison to non-hypertensive men. This association was weaker when estimating prevalence ratios (PRs) assessed with Poisson regression (PR 7.31, 95\% CI 0.96 to 55.6, data not shown). Nevertheless, the prevalence of hypertension is high in elderly men, $74.2 \%$ of German men between 70 and 79 years and $59.8 \%$ between 60 and 69 years suffer from hypertension or take antihypertensive drugs. ${ }^{27}$ However, men with prevalent conditions such as hypertension cannot be excluded from medical surveillance. This supports the use of calretinin as an additional marker, as it was not affected by hypertension.

No influence of age could be observed for calretinin, confirming the initial results by Raiko $e t a l .^{5}$ In contrast, a weak influence of age on mesothelin could be observed but was only marginally significant when estimating PRs (data not shown). Published results are inconsistent: whereas Pass et al noted no differences of mesothelin concentrations regarding age, ${ }^{28}$ more recent studies suggested that age was a statistical predictor of mesothelin. ${ }^{1029}$ In this study, we confirm these results analysing 
mesothelin concentration in plasma samples of a large number of people from the general population. Thus, for the assessment of mesothelin results age needs to be considered in diagnostics, for example, using an age-depended cut-off. As calretinin is not influenced by age, this also supports calretinin as an appropriate additional marker in early detection.

It was suggested that the release of mesothelin into serum results from malignant mesothelium and not from other inflammatory or malignant pulmonary or pleural diseases. ${ }^{30}{ }^{31}$ In this study, bronchitis was marginally associated with increased mesothelin concentrations. However, bronchial asthma was not associated with increased mesothelin but calretinin concentrations. The connection between inflammatory diseases and increased mesothelin and calretinin values in human plasma remains unclear. However, these findings are based on small numbers with 11 men with diagnosed bronchitis and 18 men with diagnosed bronchial asthma. Additional studies comprising higher numbers of these diseases are needed to assess the influence on the marker concentrations in more detail. However, in screening inflammatory indices should be recorded during medical examinations. ${ }^{20}$

Cancer was diagnosed in 20 participants after blood collection, for nine subjects already within 12 months. A sensitivity analysis revealed that cancer closer to blood collection had a stronger impact on calretinin concentrations than cancers detected later than 1-year past blood collection (data not shown). This is in agreement with a study on bladder cancer ${ }^{32}$ and on ovarian cancer, ${ }^{33}$ where cancer-related biomarkers showed more frequently elevated concentrations exceeding cut-offs 1 year prior to diagnosis. Four participants with cancer past blood collection showed increased calretinin concentrations above the cut-off at time of blood collection. As none of the identified predictors (renal dysfunction or bronchial asthma) seemed to be responsible for the elevated calretinin concentration, the later developed cancer might be responsible for the increased calretinin concentrations in the plasma of these men. However, as the types of cancers were diverse (prostate adenocarcinoma, renal cell carcinoma, urothelial tumour of the renal pelvis and basal cell carcinoma) prospective studies are needed to analyse the feasibility of calretinin as marker for the early detection of cancers other than mesothelioma.

\section{CONCLUSIONS}

Mesothelin and calretinin showed high specificities in this cohort of cancer-free elderly men. Mesothelin was strongly affected by renal dysfunction and at a lower degree by hypertension, where cystatin C may serve as an informative parameter to improve specificity. Calretinin positivity was also affected by renal dysfunction-but to a lesser extent-and showed no association with hypertension. Calretinin might be a useful adjunct to mesothelin for the early detection of mesothelioma, with an increase of the specificity to $99.1 \%$ (95\% CI $97.9 \%$ to $99.7 \%$ ) for this marker panel.

Contributors SC, DGW, GJ, DT, SM, K-HJ, TB and BP conceived and designed the experiments and analyses. IR and CM performed the experiments. SC analysed the data. SC and DGW drafted the manuscript. All authors critically revised and approved the final version of this paper.

Funding The Institute of Medical Informatics, Biometry and Epidemiology was supported by the German Social Accident Insurance grant number FP 295.

Disclaimer The authors from the Institute for Prevention and 0ccupational Medicine of the German Social Accident Insurance, Ruhr-Universität Bochum (IPA) are independent from the German Social Accident Insurance in study design, access to the collected data, responsibility for data analysis and interpretation, and the right to publish. The views expressed in this paper are those of the authors and not necessarily those of the German Social Accident Insurance.

Competing interests None declared.

Patient consent Obtained.

Ethics approval Ethical commission of the Medical Faculty of the University Duisburg-Essen (approval number 11-4678).

Provenance and peer review Not commissioned; externally peer reviewed. Data sharing statement № additional data are available.

Open Access This is an Open Access article distributed in accordance with the Creative Commons Attribution Non Commercial (CC BY-NC 4.0) license, which permits others to distribute, remix, adapt, build upon this work non-commercially, and license their derivative works on different terms, provided the original work is properly cited and the use is non-commercial. See: http://creativecommons.org/ licenses/by-nc/4.0/

(C) Article author(s) (or their employer(s) unless otherwise stated in the text of the article) 2017. All rights reserved. No commercial use is permitted unless otherwise expressly granted.

\section{REFERENCES}

1. Robinson BM. Malignant pleural mesothelioma: an epidemiological perspective. Ann Cardiothorac Surg 2012;1:491-6.

2. Hodgson JT, McElvenny DM, Darnton AJ, et al. The expected burden of mesothelioma mortality in Great Britain from 2002 to 2050. Br J Cancer 2005;92:587-93.

3. Cui A, Jin XG, Zhai K, et al. Diagnostic values of soluble mesothelinrelated peptides for malignant pleural mesothelioma: updated metaanalysis. BMJ Open 2014;4:e004145.

4. Ordóñez NG. Value of calretinin immunostaining in diagnostic pathology: a review and update. Appl Immunohistochem Mol Morphol 2014;22:401-15.

5. Raiko I, Sander I, Weber DG, et al. Development of an enzyme-linked immunosorbent assay for the detection of human calretinin in plasma and serum of mesothelioma patients. BMC Cancer 2010;10:242.

6. Johnen G, Gawrych K, Raiko I, et al. Calretinin as a blood-based biomarker for mesothelioma. BMC Cancer 2017;17:386.

7. Pesch B, Brüning $\mathrm{T}$, Johnen $\mathrm{G}$, et al. Biomarker research with prospective study designs for the early detection of cancer. Biochim Biophys Acta 2014;1844:874-83.

8. Beyer HL, Geschwindt RD, Glover CL, et al. MESOMARK: a potential test for malignant pleural mesothelioma. Clin Chem 2007;53:666-72.

9. Hollevoet K, Bernard D, De Geeter F, et al. Glomerular filtration rate is a confounder for the measurement of soluble mesothelin in serum. Clin Chem 2009;55:1431-3.

10. Weber DG, Johnen G, Taeger D, et al. Assessment of confounding factors affecting the tumor markers SMRP, CA125, and CYFRA21-1 in serum. Biomark Insights 2010;5:1-8.

11. Casjens S, Pesch B, Robens S, et al. Associations between former exposure to manganese and olfaction in an elderly population: results from the heinz nixdorf recall study. Neurotoxicology 2017;58:58-65.

12. Schmermund A, Möhlenkamp S, Stang A, et al. Assessment of clinically silent atherosclerotic disease and established and novel risk factors for predicting myocardial infarction and cardiac death in healthy middle-aged subjects: rationale and design of the Heinz Nixdorf RECALL Study. Am Heart J 2002;144:212-8. 
13. Weber DG, Taeger D, Pesch B, et al. Soluble mesothelin-related peptides (SMRP) - high stability of a potential tumor marker for mesothelioma. Cancer Biomark 2007;3:287-92.

14. Eisele L, Dürig J, Broecker-Preuss M, et al. Prevalence and incidence of anemia in the German Heinz Nixdorf Recall Study. Ann Hematol 2013;92:731-7.

15. Zalcman G, Mazieres J, Margery J, et al. Bevacizumab for newly diagnosedpleural mesothelioma in the Mesothelioma Avastin Cisplatin Pemetrexed Study(MAPS): a randomised, controlled, openlabel, phase 3 trial. Lancet 2016;387:1405-14.

16. Bertoglio P, Ambrogi MC, Chella A, et al. Is less also better? A singleinstitution experience on treatment of early stage Malignant Pleural Mesothelioma. Eur J Surg Oncol 2017;43:1365-71.

17. Dozier J, Zheng H, Adusumilli PS. Immunotherapy for malignant pleural mesothelioma: current status and future directions. Trans/ Lung Cancer Res 2017;6:315-24.

18. Scherpereel A. Malignant pleural mesothelioma: newtreatments, new hopes? Eur Respir J 2017;49:1700319.

19. Wu L, de Perrot M, de PM. Radio-immunotherapy and chemoimmunotherapy as a novel treatment paradigm in malignant pleural mesothelioma. Trans/ Lung Cancer Res 2017;6:325-34.

20. Tsim S, Kelly C, Alexander L, et al. Diagnostic and Prognostic Biomarkers in the Rational Assessment of Mesothelioma (DIAPHRAGM) study: protocol of a prospective, multicentre, observational study. BMJ Open 2016;6:e013324.

21. Helland Å, Solberg S, Brustugun OT. Incidence and survival of malignant pleural mesothelioma in Norway: a population-based study of 1686 cases. J Thorac Oncol 2012;7:1858-61.

22. Lehnert $\mathrm{M}$, Kraywinkel $\mathrm{K}$, Heinze $\mathrm{E}$, et al. Incidence of malignant mesothelioma in Germany 2009-2013. Cancer Causes Control 2017;28:97-105.
23. Wolf AS, Richards WG, Tilleman TR, et al. Characteristics of malignant pleural mesothelioma in women. Ann Thorac Surg 2010;90:949-56.

24. Filler G, Bökenkamp A, Hofmann W, et al. Cystatin C as a marker of GFR--history, indications, and future research. Clin Biochem 2005;38:1-8.

25. Ma Y, Li Q, Wang J, et al. Cystatin C, a novel urinary biomarker for sensitive detection of acute kidney injury during haemorrhagic fever with renal syndrome. Biomarkers 2010;15:410-7.

26. Ritz E. Hypertension and kidney disease. Clin Nephrol 2010;74:S39-43.

27. Neuhauser H, Thamm M, Ellert U. Blood pressure in Germany 20082011: results of the German Health Interview and Examination Survey for Adults (DEGS1). Bundesgesundheitsblatt Gesundheitsforschung Gesundheitsschutz 2013;56:795-801.

28. Pass HI, Wali A, Tang N, et al. Soluble mesothelin-related peptide level elevation in mesothelioma serum and pleural effusions. Ann Thorac Surg 2008;85:265-72.

29. Felten MK, Khatab K, Knoll L, et al. Changes of mesothelin and osteopontin levels over time in formerly asbestos-exposed power industry workers. Int Arch Occup Environ Health 2014;87:195-204.

30. Robinson BW, Creaney J, Lake R, et al. Mesothelin-family proteins and diagnosis of mesothelioma. Lancet 2003;362:1612-6.

31. Davies HE, Sadler RS, Bielsa S, et al. Clinical impact and reliability of pleural fluid mesothelin in undiagnosed pleural effusions. Am J Respir Crit Care Med 2009;180:437-44.

32. Bonberg N, Pesch B, Behrens T, et al. Chromosomal alterations in exfoliated urothelial cells from bladder cancer cases and healthy men: a prospective screening study. BMC Cancer 2014;14:854.

33. Blyuss O, Gentry-Maharaj A, Fourkala E-O, et al. Serial patterns of ovarian cancer biomarkers in a prediagnosis longitudinal dataset. Biomed Res Int 2015;2015:1-6. 